\title{
In-Situ Crystallographic Investigations of Solar System Objects in the next Decade
}

D.F. Blake ${ }^{1}$, R.M. Hazen ${ }^{2}$, S.M. Morrison ${ }^{2}$, T. S. Bristow ${ }^{1}$, P. Sarrazin ${ }^{3}$, K. Zacny ${ }^{4}$, E. B. Rampe ${ }^{5}$, R. T. Downs ${ }^{6}$, A. Yen, D. W. Ming, ${ }^{5}$ R. V. Morris ${ }^{5}$, D. T. Vaniman ${ }^{8}$, A. Treiman ${ }^{9}$, C. N. Achilles $^{10}$, P. I. Craig ${ }^{8}$, D. J. Des Marais ${ }^{1}$, V. Tu ${ }^{11}$, N. Castle ${ }^{8}$, and M. T. Thorpe ${ }^{5}$

${ }^{1}$ Exobiology Branch, MS 239-4, NASA Ames Research Center, Moffett Field, CA USA (david.blake@nasa.gov; 650-604-4816);

${ }^{2}$ Carnegie Institution for Science, Washington, DC USA;

${ }^{3}$ ExaminArt LLC, Mountain View, CA USA;

${ }^{4}$ Honeybee Robotics, Pasadena, CA USA;

${ }^{5}$ Astromaterials Research and Exploration Science Division, NASA Johnson Space Center Houston, TX USA;

${ }^{6}$ Department of Geosciences, Univ. of Arizona, Tucson, AZ USA;

${ }^{7}$ Jet Propulsion Laboratory, Pasadena, CA USA;

${ }^{8}$ Planetary Science Institute, Tucson, AZ USA;

${ }^{9}$ Lunar and Planetary Institute, Houston, TX USA;

${ }^{10}$ NASA Goddard Space Flight Center, Greenbelt, MD USA;

${ }^{11}$ Jacobs/JETS at NASA Johnson Space Center, Houston, TX USA; 


\section{In Situ Crystallographic Investigations of Solar System Objects in the next Decade}

\section{The Case for In Situ Crystallographic Investigations and Instruments}

The history of solid solar system materials, whether planetary bodies, planetary satellites, or icy or rocky planetesimals, is written in the rocks and the minerals they contain. Minerals provide the most persistent, accessible, and information-rich record of the origin(s) and evolution of solar system objects, because minerals are stable under a wide range of pressure, temperature, and composition (PTX). Regolith material comprised of specific minerals can be used to identify the conditions under which it formed, as well as subsequent environmental changes, based on individual mineral stability ranges, complex mineral assemblages, and the presence or absence of equilibrium among them. More than elemental, isotopic or optical analysis, definitive and quantitative mineralogical analysis with X-ray Diffraction (XRD) is a direct and unambiguous crystallographic technique. Fully quantitative mineralogical results are obtained from XRD patterns using Rietveld refinement and other full-pattern fitting techniques [1-3]. XRD provides detailed information about history, environment, and habitability: Past and present climates, the changing activity of water, and the availability and chemical state of biologically significant elements, all through the lens of deep time. Such quantitative mineralogical determinations are not possible if the complete mineral assemblage is not characterized. Chemical, optical, calorimetric or oxidation state data are seldom definitive because any particular chemical compositions, optical emission/absorption features, calorimetric data or valence information can still represent a range of substances or mineral assemblages.

Mineral "species" are defined as naturally occurring crystalline thermodynamic phases that have a unique combination of atomic structure and chemical composition. More than 5,500 different mineral species have been described on Earth and their crystallographic structures and other characteristics are tabulated in the open-access ICDD and RRUFF databases [4-5] among others. In addition, about 400,000 crystalline inorganic compounds have been characterized and would be classified as minerals if they were found in the natural environment. Statistical studies reveal that thousands of minerals are as yet undiscovered on Earth and throughout the Solar System [6-7]. If an unknown phase (or an inorganic compound not found as a mineral on Earth) is identified by itself or in a simple association, it can be fully characterized (both structure and composition), and its significance to the geologic history of its parent body understood by XRD because, as a crystallographic technique, XRD relies on first principles of symmetry and atomic arrangement for its determinations.

The comprehensive, detailed characterization of the mineralogy of any solar system body is fundamental to understanding its origins and evolution. "Mineral evolution," the study of the diversity and distribution of minerals through deep time [8-9], reveals that planets and moons experience a sequence of mineralogical stages, each a response to varying physical, chemical, and (in the case of Earth) biological processes. Minerals provide the most robust testimony for such critical aspects of planetary evolution as the differentiation of core, mantle, and crust; the establishment of a geodynamo; the nature and extent of volcanism; the initiation of plate tectonics; the presence and dynamic history of a hydrosphere; the evolution of the atmosphere; and the origin and evolution of a biosphere. Only minerals can preserve such history over billions of years. Only with a comprehensive documentation of mineral species, including detailed information on their structural states and compositional idiosyncrasies by XRD, can we hope to fully document that rich history. 


\section{CheMin: The first crystallographic instrument to analyze rocks and soil on another planet}

The CheMin instrument on the MSL rover Curiosity [10] determines the mineralogy and elemental composition of powdered Martian regolith using XRD. As the first crystallographic instrument flown in space, CheMin's mineralogical analyses have been critical to a number of first-of-their-kind achievements during its 8 year deployment on Mars. CheMin data provided the first quantitative mineralogical analysis of Mars soil [11-12], the first identification and characterization of an ancient habitable environment on Mars [13-15], established a maximum limit on the $\mathrm{CO}_{2}$ content of the Mars atmosphere in Hesperian time [16] and provided the first direct evidence of silicic volcanism on Mars [17], the first ground-truth mineralogy of orbital spectral measurements [18], and the first in situ evidence of the gradual drying out and oxidation of the Gale crater environment (and by proxy the early Mars atmosphere) [19]. All of CheMin's mineralogical analyses have been of basaltic composition soils, sandstones and mudstones and their alteration products, similar to mineralogies expected on the rocky planets and planetesimals.

The methodology by which a mineral's refined lattice parameters are used to deduce its elemental composition, and elemental compositions and mineralogies of regolith samples analyzed during the first 5 years of CheMin's deployment on Mars are reported in Morrison et al. [20-21]. All of the data collected by CheMin during its 8-year deployment on Mars, as well as open access papers can be found in [22] and in a review article [23] summarizing the first 6 years of mineralogical results from Mars. As summarized by Velbel in a "Highlights and Breakthroughs" article in American Mineralogist ("Crystallography: Curiosity's Bragging Right”) [24]:

"....The mineral abundances and compositions determined from MSL Curiosity CheMin data are the most complete mineralogical data set for Mars surface materials until a Mars Sample Return mission (MSR) which is still at least a decade away."

Quantitative mineralogic analysis with XRD will significantly enhance the science return of any landed mission. Moreover, there are a number of solar system objects for which the deployment of XRD instrumentation should be a scientific requirement:

- Planets for which sample return is not feasible in the forseeable future (Mercury, Venus);

- Planets and satellites for which sample return is feasible, but for which returned samples can't represent the full mineralogical diversity of their surfaces (Mars, the Earth's moon);

- Planets and other solar system objects for which it is not technologically possible to return samples in their pristine state (icy planesimals, Mars [25]).

\section{The next generation of spacecraft-capable crystallographic instruments}

Next-generation XRD instruments benefit from a decade of post-CheMin technology development, yielding a $>10 \mathrm{X}$ increase in data acquisition speed, a $50 \%$ reduction in mass and volume, and improved pattern resolution. It is now possible in a spacecraft-capable XRD to:

- Identify all minerals present $>1 \%$

- Quantify all minerals $>3 \%$ and determine their structure states

- Quantify all major elements present in each mineral ( $\mathrm{H}$ and above)

- Determine the valence state of all major elements ( $\mathrm{H}$ and above), including speciation of multi-valent elements such as Fe.

- Determine the amount of X-ray amorphous material (if present).

There are no instruments currently in NASA's planetary inventory (other than CheMin) that can claim even one of these capabilities. 


\section{Example quantitative mineralogical analysis with "Terra" a Commercial Spinoff of CheMin}

A powdered basalt sample from Hawai'i was analyzed in a Terra XRD/XRF [26] for 15 minutes, then reanalyzed for 8 hours in a Rigaku laboratory XRD. Table 1 shows the resulting Rietveld refinement and quantitative analysis from Terra, compared to that of the Rigaku XRD. Compositions (formulae) for minerals having variable chemistry (andesine, augite, pigeonite and forsterite) are derived from lattice parameters [20] obtained with Rietveld refinement [1] of the Terra XRD pattern. Clay minerals and the amorphous component were quantified using FULLPAT [2,3].

Next-generation CheMin instruments with optimized Terra diffraction geometry will provide a dataset equivalent to that shown in Table 1 in one hour or less.

Table 1. Quantitative Analysis of a Hawai'ian Basalt Cobble

\begin{tabular}{|c|c|c|c|}
\hline Phase & Formula & Wt.\% & Wt.\% \\
\hline Andesine $^{1}$ & $\mathrm{Ca}_{0.24} \mathrm{Na}_{0.26}\left(\mathrm{Al}_{0.735} \mathrm{Si}_{3.265}\right) \mathrm{O}_{8}$ & 34.9 & 27.9 \\
\hline Augite $^{1}$ & $\mathrm{Mg}_{0.82} \mathrm{Fe}_{0.52} \mathrm{Ca}_{0.66} \mathrm{Si}_{2} \mathrm{O}_{6}$ & 15.4 & 19.9 \\
\hline Pigeonite $^{1}$ & $\left(\mathrm{Mg}_{0.54} \mathrm{Fe}_{0.46}\right) \mathrm{SiO}_{3}$ & 10.8 & 13.3 \\
\hline Forsterite $^{1}$ & $\left(\mathrm{Mg}_{0.69} \mathrm{Fe}_{0.31}\right) \mathrm{SiO}_{4}$ & 7.3 & 7.5 \\
\hline Ilmenite & $\mathrm{FeTiO}_{3}$ & 0.8 & 1.5 \\
\hline Hematite & $\mathrm{Fe}_{2} \mathrm{O}_{3}$ & 0.3 & 0.4 \\
\hline Magnetite & $\mathrm{Fe}_{3} \mathrm{O}_{4}$ & ND & 1.1 \\
\hline Cristobalite & $\mathrm{SiO}_{2}$ & 5.1 & 2.6 \\
\hline Amorphous $^{2}$ & & 20.3 & 20.9 \\
\hline Nontronite $10.0^{2}$ & & 4.4 & 4.0 \\
\hline Total & & 99.3 & 99.1 \\
\hline
\end{tabular}

${ }^{1}$ Elemental composition determined from refined lattice parameters [20]

${ }^{2}$ Quantified using the FULLPAT method [2]

\section{Deployment of crystallographic instruments in the solar system.}

Mercury. The MESSENGER mission revealed that Mercury is a geochemical end-member among the terrestrial planets, and as such, an in-situ surface investigation of its mineralogy and chemistry will greatly aid our understanding of planet formation and planetary evolution. Mercury is the most chemically reduced of the terrestrial planets, diverse in terms of surface compositions, and volatile rich [27] and references therein. Upcoming orbital observations by BepiColombo will reveal its surface composition and other features in much greater detail [28]. However, the mercurian surface lacks diagnostic spectral absorption features in the UV-VIS region, so the mineralogy of Mercury has only been inferred through normative calculations based on surface compositions, and uncertainties in the oxygen content of the surface result in a large range of possible minerals that could be present [29-31]. The deployment of a crystallographic instrument such as next-generation CheMin [32] could with definitive mineralogical analysis, revolutionize our understanding of the geologic history of that planet and of comparative terrestrial planetology. A simplified lander equipped with a Honeybee Robotics PlanetVac system [33] (Fig. 1) could deliver powdered regolith to a next generation XRD instrument such as shown in Fig. 2 [32]. The instrument has no moving parts and collects XRD patterns from two separate samples delivered to it. More capable instruments suitable for four [34] or many [35] analyses are possible.

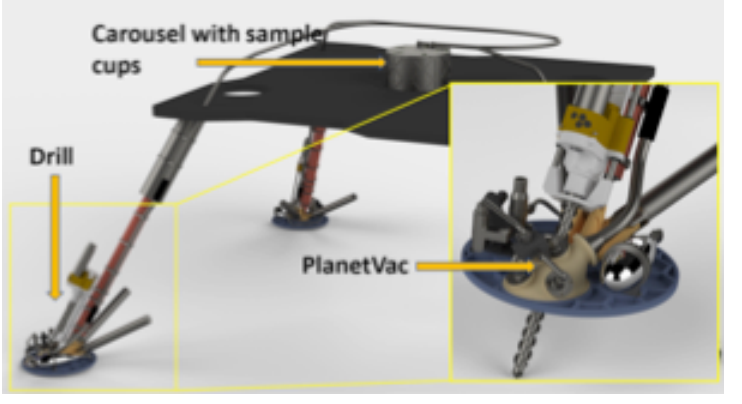

Fig. 1: PlanetVac moves a regolith sample from a lander footpad to instruments inside the lander pneumatically, with no requirement for an arm or complicated moving parts. 

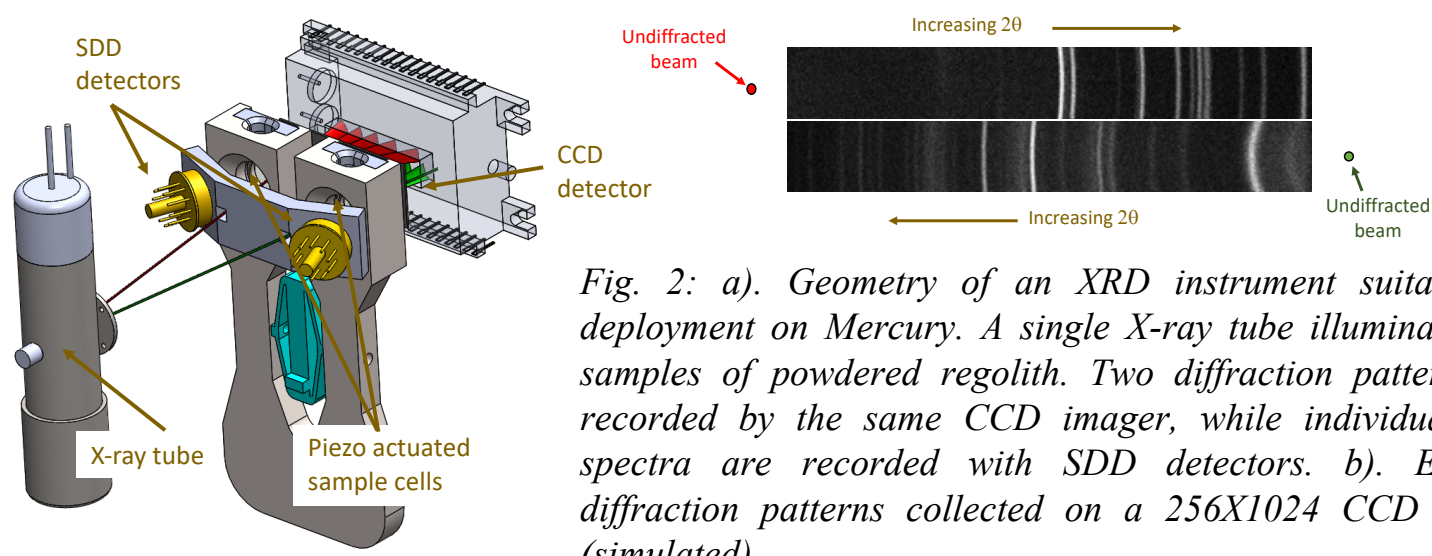

Fig. 2: a). Geometry of an XRD instrument suitable for deployment on Mercury. A single X-ray tube illuminates two samples of powdered regolith. Two diffraction patterns are recorded by the same CCD imager, while individual XRF spectra are recorded with SDD detectors. b). Example diffraction patterns collected on a $256 X 1024$ CCD imager (simulated).

Venus. Quantitative and definitive mineralogy is critical for elucidating the early history and evolution of Venus, for deducing its early habitability, for comparative planetology of the rocky planets (Mercury, Venus, Earth, Mars), and for characterizing Venus as a surrogate for Venus-like exoplanets. Principal goals of Venus exploration are listed in [36]. The goals for inner planet research in the present decade include [37]:

- Understanding the origin and diversity of terrestrial planets.

- Understanding how the evolution of terrestrial planets enables and limits the origin and evolution of life.

- Understanding the processes that control climate on Earth-like planets.

An XRD instrument suitable for a landed Venus mission is described in a Decadal Survey white paper [34], and is included in the baseline payload of a Venus Flagship mission study submitted to the Decadal Survey [38].

Mars. The Mossbauer spectrometers on Mer-A and Mer-B identified and quantified the iron minerals in Mars soil and rocks [39], but to date fully quantitative mineralogy of the Martian regolith has only been determined in Gale crater by the CheMin instrument. Mars 2020 will be deployed to Jezero crater, a deltaic system preserved in an ancient crater lake (a locality that is infamily with Gale crater); however quantitative mineralogical analyses of these samples will have to await sample return.

Mars is a topographically, geologically and mineralogically diverse planet. Mars landing site selection workshops for MER, MSL and Mars 2020 have identified dozens of scientifically valuable sites worthy of investigation [40-42]. To fully understand the mineralogical diversity and geologic history of Mars for comparative terrestrial planetology, for Astrobiology, for In Situ Resource Utilization (ISRU) and for planning future human exploration and colonization, many sites must be fully characterized using definitive and quantitative mineralogical techniques. An XRD instrument suitable for inclusion in the payload of a MER-class rover is described in a Decadal Survey white paper [35].

Earth's Moon. The mineralogical composition of lunar soil can be used to elucidate its petrogenesis and that of its parental lithologies (e.g., igneous rocks, impact breccias), as well as subsequent diagenetic or metamorphic events. In addition to its value to landed lunar science and as ground truth for orbital missions, in-situ mineralogical analysis can be used to:

- Evaluate potential In Situ Resource Utilization (ISRU) processes;

- Discover ore deposits useful for extraction of rare earth or other valuable elements; 
- Characterize the polar environment including exotic volatile-rich materials generated in permanent shadow and transported to the local site by impacts (e.g., clays or iron hydroxides);

Taylor et al. [43] report the mineralogy of 118 returned Apollo regolith samples in the $<150$ $\mu \mathrm{m}$ grain-size range analyzed by Terra. Sun et al. [44] report XRD-based ground-truth mineralogy of the Apollo 17 landing site. XRD patterns and mineral abundances from [43] are available on the Open Data Repository [45].

Rocky and Icy Planetesimals. Rocky and icy planetesimals are the most primitive objects in the solar system and hold clues to its formation and that of portions of the pre-solar nebula.

Many but not all rocky planetesimals (asteroids) are well represented in meteorite collections on Earth. However, some, such as olivine rich "mantle" asteroids are underrepresented and as a result poorly understood. Absent sample return missions, in-situ XRD analysis is perhaps the only way to understand these and other rocky planetesimals too small, too diverse, too friable or too rare to be represented in terrestrial collections.

Icy Planetesimals (cometary nuclei, Kuiper belt / Oort cloud objects) have long been considered key to understanding the processing and delivery of water and organics to the early solar system. Comet nucleus flybys, rendezvous, and even landings (e.g., Rosetta[46]) have provided a wealth of new information. However, even (planned) Comet Nucleus Sample Return missions will be incapable of returning cometary regolith in its pristine condition. The icy minerals in these objects retain the same P,T,X information and history as do their counterparts in rocky planetesimals, but their thermal stability does not extend into the temperature ranges proposed for sample return (changes in water ice and solid gases can occur as low as $\sim 30$ kelvins [47]). Low-pressure water ice can exist in at least two amorphous states ("high density" and "low density"), and two crystalline states (cubic and hexagonal) [47]. In addition, gases such as $\mathrm{CO}, \mathrm{CH}_{4}, \mathrm{CH}_{3} \mathrm{OH}$ and others can be contained in type I and type II water ice clathrates (possibly explaining the anomalous gas releases during apparations [48]). We will not know the lowtemperature petrology of these objects (or understand how they were formed or processed in the early solar nebula) until in-situ XRD analyses are performed by a icy planetsimal lander.

\section{Summary}

The history of solar system materials, whether planetary bodies, planetary satellites, or icy or rocky planetesimals, is written in the rocks and the minerals they contain. Due to their stability under a wide range of pressure, temperature, and composition, minerals provide the most persistant, accessible, and information-rich record of the origin(s) and evolution of solar system objects. To fully document the rich history of our solar system and our universe requires comprehensive analysis and documentation of mineral species, including detailed information on their structural states and compositional idiosyncrasies by XRD.

\section{References}

[1]. Bish, D.L. and J.E. Post (1993). "Quantitative mineralogical analysis using the Rietveld fullpattern fitting method." Am. Min., 78, 932-940.

[2] Chipera, S.J. and D.L. Bish (2002). "FULLPAT: A full-pattern quantitative analysis program for X-ray powder diffraction using measured and calculated patterns." J. Applied Crystallography 35, 744-749.

[3]. Chipera, S.J., \& D.L. Bish (2013). "Fitting full X-ray diffraction patterns for quantitative analysis: a method for readily quantifying crystalline and disordered phases." Advances in Materials Physics and Chemistry, 3(01), 47. 
[4] Lafuente B., R.T. Downs, H. Yang, N. Stone (2015). "The power of databases: the RRUFF project." In: Highlights in Mineralogical Crystallography, T Armbruster and R M Danisi, eds. Berlin, Germany, W. De Gruyter, pp 1-30.

[5] https://www.icdd.com/pdfsearch/

[6]. Hazen, R.M., et al. (2015). "Mineral ecology: Chance and necessity in the mineral diversity of terrestrial planets." Canadian Mineralogist, 53, 295-323.

[7]. Hystad, G., et al. (2019). "Bayesian estimation of Earth's undiscovered mineralogical diversity using noninformative priors." Mathematical Geosciences, 51, 401-417.

[8]. Hazen, R.M., et al. (2008). "Mineral evolution.” American Mineralogist, 93, 1693-1720.

[9]. Hazen, R.M. (2012). “The Story of Earth: The First 4.5 Billion Years, from Stardust to Living Planet." New York: Viking, 306 p.

[10] Blake, D.F., et al. (2012). "Characterization and Calibration of the CheMin Mineralogical Instrument on Mars Science Laboratory." Space Science Reviews, Vol. 170, Issue 1-4, pp. 341-399.

[11]. Blake, D.F., et al. (2013). "Curiosity at Gale crater, Mars: Characterization and analysis of the Rocknest sand shadow." Science, 341, 1239505; doi: 10.1126/science.1239505.

[12]. Bish, D.L., et al. (2013). "X-Ray Diffraction Results from Mars Science Laboratory: Mineralogy of Rocknest Aeolian Bedform at Gale crater." Science, 341, 1238932; doi: $10.1126 /$ science. 1238932

[13]. Vaniman, D.T., et al. (2014). "Mineralogy of a Mudstone on Mars." Science, $10.1126 /$ science. 1243480 .

[14]. Grotzinger, J.P. et al. (2013). "A Habitable Fluvio-Lacustrine Environment at Yellowknife Bay, Gale Crater, Mars.” Science, 10.1126/science.1242777.

[15]. Bristow, T.F., et al. (2015). "The origin and implications of clay minerals from Yellowknife Bay, Gale crater, Mars.” Am. Min. Vol. 100, pages 824-836, doi.org/10.2138/am-2015.

[16]. Bristow,T.F., et al. (2017). "Low Hesperian $\mathrm{P}_{\mathrm{CO} 2}$ from in situ mineralogical analysis at Gale crater, Mars.” PNAS 114 No. 9, pp. 2166-2170. www.pnas.org/cgi/doi/10.1073/ pnas.1616649114.

[17]. Morris, R.V., et al. (2016). "Silicic volcanism on Mars evidenced by tridymite in high-SiO ${ }_{2}$ sedimentary rock at Gale crater." PNAS: doi: 10.1073/pnas.1607098113.

[18]. Achilles, C.N., et al. (2017). "Mineralogy of an active eolian sediment from the Namib dune, Gale crater, Mars,” J. Geophys. Res. Planets, 122, doi:10.1002/2017JE005262.

[19]. Bristow, T.F., et al. (2018). "Clay Mineral Diversity and Abundance in Sedimentary Rocks of Gale Crater, Mars." Science Advances, Sci. Adv. 2018;4: eaar3330

[20]. Morrison, S.M., et al. (2018). "Relationships between unit-cell parameters and composition for rock-forming minerals on Earth, Mars, and other extraterrestrial bodies," American Mineralogist. DOI: 10.2138/am-2018-6123.

[21]. Morrison, S.M., et al. (2018). "Crystal chemistry of martian minerals from Bradbury Landing through Naukluft Plateau, Gale crater, Mars," American Mineralogist. DOI: 10.2138/am2018-6124.

[22]. http://odr.io/CheMin

[23]. Rampe, E.B., et al. (2020). https://doi.org/10.1016/j.chemer.2020.125605. 
[24]. Velbel, M.A. (2018). DOI: http://doi.org/10.2138/am-2018-6468CCBYNCND

[25]. Vaniman, D.T. et al. (2018). DOI: http://doi.org/10.2138/am-2018-6346

[26]. Sarrazin, P., et al. (2008). "Field studies of Mars analog materials using a portable XRD/XRF instrument." LPS XXXIX, abstr\# 2421.

[27]. Armytage, R.M. et al., (2018). https://www.researchgate.net/publication/329504378.

[28]. Benkhoff, J. et al., (2010). DOI: 10.1016/j.pss. 2009.09.020.

[29]. Vander Kaaden, K.E., et al., (2017), Icarus 285, 155-168.

[30]. McCubbin, F.M., et al., (2017), J.G. Planets 122, 2053-2076.

[31]. Namur, O. and B. Charlier (2017), Nature Geosci. 10, 9-13.

[32]. Blake, D.F. et al. (2020). "In Situ Mineralogical Analysis of Mecurian Regolith using X-ray Diffraction and X-ray Fluorescence.” LPSC 50, Abstr. \#1808.

[33]. Zacny, K. and A. Garcia, (2014). IEEE Aerospace Conference, 3-7 Mar. 2014, Big Sky MT.

[34]. Blake, D.F., T.F. Bristow, P. Sarrazin and K. Zacny (2020). "In-Situ Mineralogical Analysis of the Venus Surface using X-ray Diffraction.” Decadal Survey White Paper.

[35]. Rampe, E.B. et al. (2020). "CheMinX: A Next Generation XRD/XRF for Quantitative Mineralogy and Geochemistry on Mars." Decadal Survey White Paper.

[36]. Scientific Goals, Objectives and Investigations for Venus Exploration (2019).

[37]. Visions and Voyages for Planetary Science in the Decade 2013-2022.

[38]. Gilmore, M. et al. (2020). "Venus Flagship Mission Study." (NNH18ZDA001N-PMCS); submitted to the Decadal Survey for the 2023-2033 decade.

[39]. Morris, R.V., G. Klingelhöfer and J.F. Bell (2008). Iron mineralogy and aqueous alteration on Mars from the MER Mössbauer spectrometers. The Martian Surface: Composition, mineralogy, and physical properties. Cambridge Univ. Press, UK, 339-365.

[40]. Grant, J.A., et al. (2010) "The science process for selecting the landing site for the 2011 Mars Science Laboratory.” Planet. Space Sci., 59, 1114-1127, doi:10.1016/j.pss.2010.06.016.

[41]. Grant, J.A., et al. (2004). "Selecting landing sites for the 2003 Mars Exploration Rovers." Planet. Space Sci., 52, 11-21, doi: 10.1016/j.pss.2003.08.011.

[42]. Grant, J.A., et al. (2018). "The science process for selecting the landing site for the 2020 Mars rover." Planetary and Space Science, doi: 10.1016/j.pss.2018.07.001.

[43] Taylor, G.J. et al. (2019), GCA 266, pp. 17-28,https://doi.org/10.1016/j.gca.2019.07.046

[44]. Sun, L. et al. (2018), LPSC 49, \#1693; AbSciCon 2017, \#3074.

[45]. https://odr.io/lunar-regolith-xrd

[46]. https://sci.esa.int/web/rosetta/

[47]. Jenniskens, P. and D.F. Blake. (1994). "Structural transitions in amorphous water ice and astrophysical implications." Science 265:753-756.

[48]. Blake, D., L. Allamandola, S. Sandford, D. Hudgins and F. Freund (1991). "Clathrate hydrate formation in amorphous cometary ice analogs in vacuo." Science, 254:548-552. 